\title{
DECOLONIZANDO ACCIONES PUBLICAS CONTRA EL FEMINICIDIO CON CUERPOS DISIDENTES: EL PERFORMANCE Y LA PLATAFORMA ARTE ACCIÓN EN CHIAPAS, MEXICO
}

\section{Artículo de Reflexión}

\section{Brittany Chávez}

Universidad de Carolina del Norte en Chapel Hill / bchavez@live.unc.edu

Brittany Chávez es artista-intelectual-militante-activista-pedagoga residente en San Cristóbal de las Casas, Chiapas [México]. Es estudiante doctoral de comunicación [enfocada en performance y estudios decoloniales] en el departamento de estudios de comunicación de la Universidad de Carolina del Norte en Chapel Hill. También es una de las integrantes principales del grupo internacional de performance La Pocha Nostra.

\section{Doris Difarnecio}

CESMECA [Centro de Investigaciones Superiores de México y Centroamérica] / dorisdifarnecio@gmail.com

Doris Difarnecio es directora teatral-intelectual-activista residente en San Cristóbal de las Casas, Chiapas, dónde dirigió el Centro Hemisférico [satélite del Instituto Hemisférico de Performance y Política en las Américas] entre 2007-2013 y FOMMA [Fortaleza de la Mujer Maya) entre 1999 y 2013. Actualmente es estudiante de maestría en CESMECA [Centro de Investigaciones Superiores de México y Centroamérica] enfocada en cuestiones de teatro popular y género en la región de Chiapas. 
CHAVEZ B, [2014]Decolonizando acciones públicas contra el feminicidio con cuerpos disidentes:

El performance y la plataforma arte acción en Chiapas México Calle14, 9 [14] página 30-43

\title{
DECOLONIZANDO ACCIONES PÚBLICAS CONTRA EL FEMINICIDIO CON CUERPOS DISI- DENTES: EL PERFORMANCE Y LA PLATAFORMA ARTE ACCIÓN EN CHIAPAS MÉXICO
}

\begin{abstract}
RESUMEN
Proponemos una forma alternativa de denunciar y visibilizar la violencia feminicida en la región de Chiapas por medio de la plataforma de performance y activismo Arte Acción. Tres partes constituyen este artículo: la situación del feminicidio en Chiapas y nuestra metodología desde lo erótico, lo decolonial y lo pedagógico; la creación de la memoria colectiva a través de acciones públicas; y nuestro trabajo continuado con la plataforma como un modo de activismo alternativo.
\end{abstract}

\section{PALABRAS CLAVES}

Performance y performatividad, memoria colectiva, pedagogía decolonial, lo erótico, acción política, feminicidio.

\section{DECOLONIZING PUBLIC ACTIONS AGAINST FEMINICIDE WITH DISSIDENT BODIES: PERFORMANCE AND THE ARTE-ACCIÓN PLATFORM IN CHIAPAS, MEXICO}

\begin{abstract}
We propose an alternative way to expose and make visible the feminicidal violence in the Chiapas region through the activism and performance platform Arte-Acción. The article is divided in three sections: the situation of feminicide in Chiapas and our methodology, based on the erotic, the decolonial and pedagogy; the creation of collective memory through public actions; and our continued work with the platform as a form of alternative activism.
\end{abstract}

\section{KEYWORDS}

Performance and performativity, collective memory, decolonial pedagogy, the erotic, political action, feminicide.

\section{DÉCOLONISATION DES ACTIONS PUBLIQUES CONTRE LE FÉMINICIDE AVEC DES ORGANISMES DISSIDENTS: LA PERFORMANCE ET LA PLATE-FORME ART ACTION AU CHIAPAS, MEXIQUE}

\section{RÉSUMÉ}

Nous proposons une autre façon d'exposer et de rendre visible la violence contre les femmes dans la région du Chiapas par la plate-forme de performance et activisme Art Action. Cet article est composé de trois parties: la situation du féminicide au Chiapas et notre méthodologie depuis l'érotisme, le décolonial et le pédagogique; la création de la mémoire collective à travers des actions publiques; et notre collaboration continue avec la plate-forme comme un mode d'activisme alternatif. 


\section{MOTS CLÉS}

Performance et performativité, mémoire collective, pédagogie décoloniale, l'érotisme, action politique, féminicide.

\section{DESCOLONIZANDO AC̣ÕES PÚBLICAS CONTRA O FEMINICÍDIO COM CORPOS DISSI- DENTES: A PERFORMANCE E A PLATAFORMA ARTE AC̣̃̃o EM CHIAPAS, MÉXICO}

\section{RESUMO}

Propomos uma forma alternativa de denunciar e visibilizar a violência feminicida na região de Chiapas por meio da plataforma de performance e ativismo Arte Ação. Três partes constituem este artigo: a situação do feminicídio em Chiapas e nossa metodologia desde o erótico, o descolonial e o pedagógico; a criação da memória coletiva através de ações públicas; e nosso trabalho continuado com a plataforma como um modo de ativismo alternativo.

\section{PALAVRAS CHAVES}

Performance e performatividade, memória coletiva, pedagogia descolonial, o erótico, ação política, feminicídio.

\section{CHIAPA MEXICOPI IAPA WARMIPA IUIAITA MANA MUNARKAKUNA TUKUIMA SUMA KAWARINGAPA}

\section{SUGLLAPI}

luianchimi willangapa mana allilla karigta, wairapa, iuiaita mana sakigtakuna kaugsachu kawachichu Chiapas, llagtapi munarikumi imasapipas kawachinga iachachingapa sugllapi apachispa chasa mana karichu.

\section{IMA SUTI RIMAI SIMI}

Performance, performatividad, sugllapi iuiai, sigpa iuiai, iachaikui, erotico, imasa kaugsai, warmipa iuiaimana tiachu. 


\section{Agradecimientos}

Agradecemos primero a los familiares de las mujeres víctimas de la violencia feminicida en la región de Chiapas [México], por su participación activa en la denuncia continua contra la violencia hacia la mujer en esta región, como parte de su proceso de duelo y resistencia contra el olvido y la ausencia de justicia por parte del Estado. También a la sociedad civil, los fotógrafos, artistas y activistas, la prensa libre, las organizaciones de derechos humanos [como Colem y Koman Ilel], quiénes han sido una parte fundamental de esta lucha. También agradecemos a Ivan Dario Vargas Rocancio y Carolina Novella por su generosidad en redacción y comentarios sobre este artículo. Para mayor información sobre las acciones detalladas en este artículo, visitar la página: <http:// arteaccionchiapasmexico.wordpress.com/>.

Es nuestra convicción y nuestra práctica que para revelarse y luchar no son necesarios ni líderes ni caudillos, ni mesías ni salvadores; para luchar solo se necesita un poco de vergüenza, un tanto de dignidad y mucha organización, lo demás o sirve al colectivo o no sirve.

De la última carta del Subcomandante Insurgente Marcos (desde el 25 de mayo, Subcomandante de Acero Inoxidable].

La memoria del trauma, la memoria del horror, es memoria en duelo; es decir, memoria cubierta de muerte pero también - o precisamente por eso

- memoria que exige justicia [Lorenzano, 2007: 12].

\section{Ubicación del feminicidio y Arte Acción en Chiapas: Cuestiones de violencia y activismo}

La región de Chiapas es políticamente reconocida, principalmente, por el zapatismo. En esta medida, la acción performátiva-pedagógica decolonial de la transformación del símbolo individual del Subcomandante Insurgente Marcos al símbolo colectivo del Subcomandante de Acero Inoxidable - luego del brutal asesinato del Subcomandante Galeano en el caracol La Realidad- el clima aquí se ha puesto aún más tenaz ${ }^{1}$. Como artistasactivistas-intelectuales formadas, en parte, por las luchas civiles en esta región donde residimos, nuestra posición geopolítica está informada y dirigida por este lugar, donde nos colocamos y nos posicionamos. Entramos a este territorio como mestizas, con humildad hacia las varias luchas presentes en esta región. Formamos parte de la sociedad civil. Este posicionamiento define nuestro lugar de enunciación.

En esta región existe una epidemia de violencia contra la mujer [bajo la forma de múltiples homicidios] que sigue aumentando, de manera totalmente impune e invisibilizada. El termino adoptado para nombrar esta violencia homicida hacia las mujeres, "feminicidio," fue empleado por la primera vez en México por Marcela Lagarde. Lagarde [1997] define feminicidio como "la política del exterminio de las mujeres" y "el conjunto de acciones que tienden a controlar y eliminar las mujeres a través del temor y el daño, obligarlas a vivir en el temor y la inseguridad, amenazadas y en condiciones humanas mínimas al negarles la satisfacción de sus reivindicaciones vitales" [Lagarde, 1997: 171]. Esta es una definición que rebasa el feminicidio de su posición regular en Ciudad Juárez. Existen varios factores que feministas como Marcela Lagarde (1997), Mercedes Olivera [2008; 2011]), Marta Figueroa [2008], Graciela Reyermuth Enciso [2008) y Rita Segato [2010] pronuncian alrededor de los casos de feminicidio, no sólo en Chiapas, sino cómo fenómeno en América Latina y en varias partes del mundo.

Mercedes Olivera [2008] atribuye el aumento de la violencia feminicida en Chiapas a "la crisis de gobernabilidad" y a "causas estructurales" como "la pobreza, el desempleo, la desestructuración

1. Esta referencia de lo performático viene de la teorista J.L. Austin: "Un performativo, para Austin [1975], refiere a situaciones en las que 'la emisión del enunciado implica la realización de una acción'” [Taylor, 2008: 31].

Pensar en el cambio de Marcos como algo 'performativapedagógica decolonial vino de una serie de conversaciones cyber con la Dra. Catherine Walsh 
de la economía campesina" y otros factores, que, para Olivera, crean profundas rupturas en el núcleo familiar [Olivera, 2008: 33]. Aquí Olivera [2008] describe el término feminicidio como parte de un sistema que desaloja e ignora los derechos humanos de la mujer. La mujer sufre la violencia no solo por parte de individuos, sino también por parte de sistemas socioeconómicos, culturales y políticos que fallan en protegerla. Graciela Reyermuth Enciso [2008] habla de la "internalización", por parte de las mujeres, de su subordinación, lo que las inhibe de emprender acciones para prevenir y cambiar la situación violenta que sufren [Reyermuth Enciso, 2008: 130]. Martha Figueroa Mier [2008] argumenta que la impunidad se naturaliza y, por consiguiente, los procesos legales ignoran el incremento de la violencia feminicida [2008: 205]. En un trabajo posterior, más cerca del momento en que escribieron las mencionadas intelectuales feministas, Marcela Lagarde [2005] se extendió sobre el feminicidio, no solamente refiriéndose a la región de Chiapas. Afirma allí que "estos crímenes se dan en todo el mundo y son el resultado de la violencia misógina llevada al extremo y por ende son la muestra más visible de múltiples formas de hostigamiento, maltrato, daño, repudio, acoso y abandono" [Lagarde, 2005: 206).

Si bien estamos de acuerdo con el trabajo de estas reconocidas académicas feministas alrededor del tema, existe un aspecto fundamental en el cual no coincidimos tanto en el campo teórico como en los terrenos práctico y político. Para nosotras, cualquier persona que se identifica a sí misma como mujer, sin consideración a su cuerpo biológico, apariencia física y/o estatus de transición física, y que muere como producto de las situaciones violentas previamente descritas, es víctima de feminicidio. En consecuencia, las transmujeres, intersex, transexuales y cualquier otro cuerpo disidente pero con identificación femenina propia, pueden ser víctimas de feminicidio. En la literatura jurídica y académica estos cuerpos no están incluidos. Inclusive, no aparecen en los medios de comunicación. En muchos casos, ni siquiera son reconocidos como homicidios porque socialmente estas personas transgreden las políticas normativas de lo que puede ser el sexo y el género de una persona. No incluir estas mujeres en casos de feminicidio constituye, en nuestra opinión, una doble violencia. De aquí en adelante, cuando nos referimos a las mujeres, la definición será la ya descrita.

Rita Segato [2010] busca una estrategia decolonial frente al feminicidio, pero persiste en la política del cuerpo biológico de la mujer. Aun así, encontramos que su gesto decolonial es importante para nuestro trabajo. Estamos de acuerdo en que parte del fenómeno feminicida, especialmente en América Latina, es el resultado de la constante producción y reproducción de la modernidadcolonialidad. Queremos reiterar lo que Segato [2010] señala: "los feminicidios, como prácticas casi maquinales de extermino de las mujeres son también una invención moderna. Es la barbarie de la colonial modernidad..." [Segato, 2010: 19]. Dicho esto, una posición decolonial se contrapone a una posición universal de derechos humanos que viene del Estado basado en esta modernidad. Para Segato [2010], la estrategia decolonial consiste no en buscar justicia por parte de los derechos humanos universales, sino por entender la pluralidad regional de cada pueblo en toda su complejidad. Caminando en conversación con Segato, nuestras acciones contra el feminicidio se distancian de estrategias estatales y movimientos políticos, para denunciar las huellas de esta violencia en espacios públicos de una manera cotidiana y participativa.

\footnotetext{
2. Es importante mencionar que nuestro uso de arte acción tiene mucha resonancia geopolítica en México. Como menciona Antonio Prieto Stambaugh, "La performance conceptual en México, también conducido como arte acción, ha experimentado diversas etapas de mutación, decadencia y renacimiento a partir de los años sesenta, cuando Alejandro Jodorowsky y Juan José Gurrola sacudieron los cimientos del teatro nacional mediante una serie de 'efímero pánicos', happenings y otros gestos antiestablishment. Desde entonces, arte acción fue el escenario idóneo para articular una corporalidad política, es decir, una corporalidad que se enfrente al poder mediante una piel transformada en pergamino diseminador de interrogantes" [2008: 609). Nuestro uso es otra mutación de la frase "arte acción" por la convergencia de arte, intervenciones públicas y la performance.
} 
Arte Acción es una plataforma con presencia digital integrada por activistas, organizaciones de derechos humanos e intelectuales ${ }^{2}$. La plataforma cruza presentaciones interdisciplinarias entre el performance, el activismo, el arte y las intervenciones urbanas contra el feminicidio en espacios públicos. Igualmente, Arte Acción está empezando a ampliarse hacia acciones de performance y política con otros temas. El énfasis reside en la construcción de iniciativas que contribuyen al debate y la acción social. La plataforma inició a raíz del feminicidio brutal de una joven indígena el 14 de Abril del 2012, Itzel Janet Méndez Pérez. Al ver que los padres estaban bajo una situación de desgarre emocional y bajo una actitud cínica e impune por parte del ministerio público en torno a la investigación, decidimos acompañar a los padres a denunciar el crimen con la primera acción de Mayo 14, 2012. Desde entonces, las acciones han sucedido en los días catorce de cada mes [con algunas excepciones por razones de participación de los familiares]. Se trata de una plataforma que continúa hasta la fecha y continuará

$\nabla$ Imagen 1. Cartel de Arte Acción; diseño: Lydia Reich.

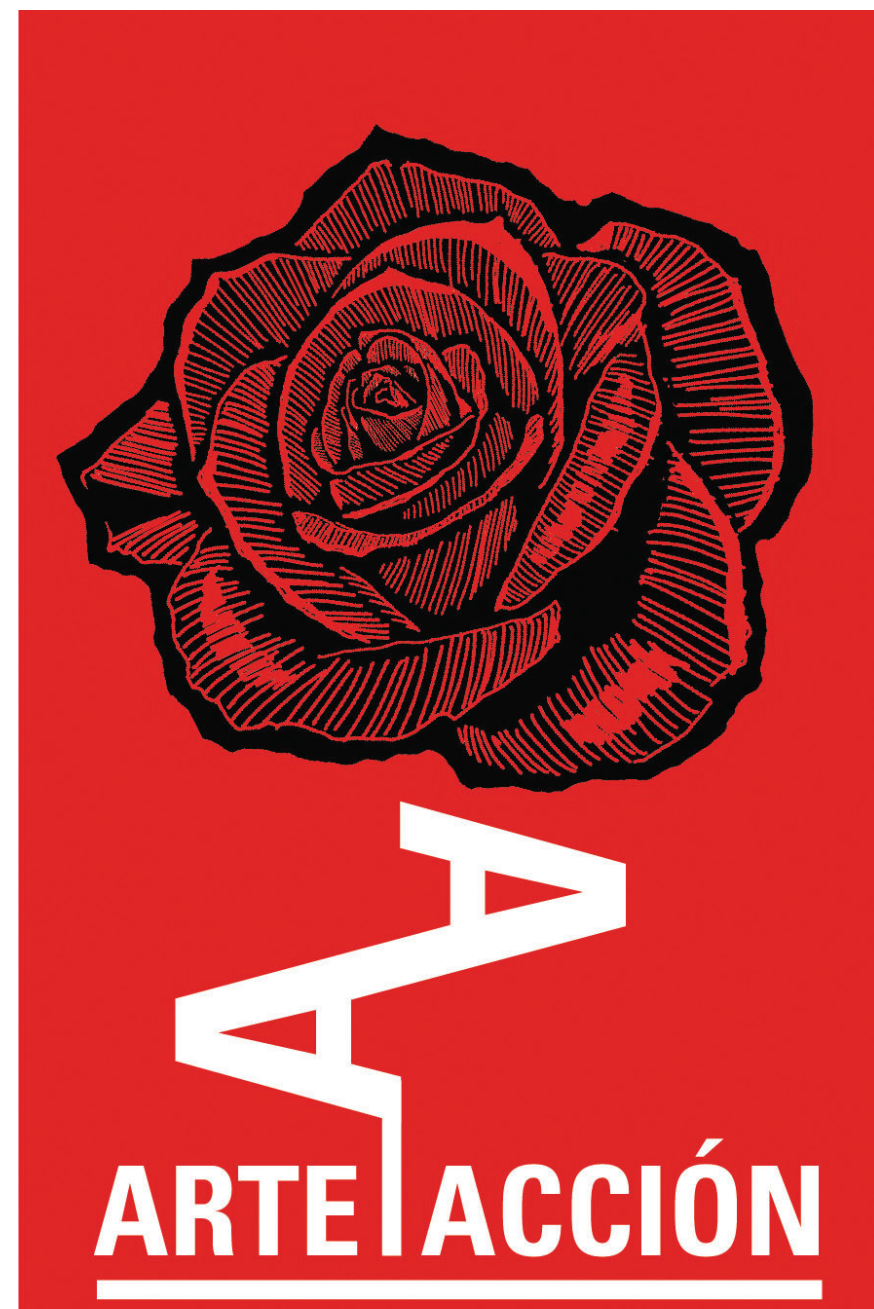

interviniendo espacios urbanos públicos.

No estamos vinculadas a ningún movimiento político amplio. Nuestras políticas vienen de la materialidad de los cuerpos presentes en el espacio público, de las acciones que dan espacio al duelo y denuncian la violencia feminicida. Las raíces de nuestras acciones tienen tres ejes principales: lo erótico, lo decolonial y lo pedagógico. Audre Lorde [1978), en un texto revolucionario, separa lo erótico y lo sexual como diametralmente opuestos. Tanto para Lorde [1978] como para nosotras lo erótico es una fuerza de vida que nos abre a las amplitudes de los sentidos y nos conecta con nuestro ser. Lo erótico nos permite profundizar en el trabajo de las acciones de una manera en que todos los cuerpos presentes tienen una conexión de vitalidad. Entrelazamos lo decolonial con lo pedagógico. De acuerdo con Catherine Walsh "[...] la decolonialidad no es una teoría por seguir sino un proyecto por asumir. Es un proceso accional para pedagógicamente andar" (Walsh, 2013: 67). La pedagogía, para Walsh -siguiendo a Friere-, no está limitada a situaciones institucionales o escolares, sino también viene de los participantes en luchas sociales de liberación que demuestran y enseñan sus aprendizajes, desaprendizajes, reaprendizajes, reflexiones y acciones [Walsh, 2013: 29]. El andar decolonial que Walsh describe es una teoría en práctica con base en las luchas sociales. Anota:

Las pedagogías pensadas así no son externas a las realidades, subjetividades e historias vividas de los pueblos y de la gente, sino parte integral de sus combates y perseverancias o persistencias, de sus luchas de concientización, afirmación y desalienación, y de sus bregas - ante la negación de su humanidad- de ser y hacerse humano. Es en este sentido y frente a estas condiciones y posibilidades vividas que propongo el enlace de lo pedagógico y lo decolonial [Walsh, 2013: 31].

El entrelace entre lo pedagógico y lo decolonial es andar con y al lado de la gente que forman parte de las luchas sociales. Desde estas posiciones surge nuestro activismo. 


\section{La memoria colectiva y el performance en acciones públicas}

Conmemorar una muerte violenta no es un acto simple. Toca profundidades de memoria y trauma que son áreas sensibles para el activismo. No se trata de reabrir una herida. Reiterar el duelo y recordar la pérdida de un ser querido recupera y politiza las emociones. También presentar el dolor de la pérdida de un espacio privado y familiar en un espacio público, convierte la memoria en resistencia y denuncia. Como dice Lorenzano [2007], "La memoria es algo activo que se sitúa en el hoy y a través del cual el pasado es permanentemente resignificado. Estamos hablando de ligar pasado, presente y futuro, no en un ejercicio de nostalgia sino en un trabajo en el que el dolor se convierte en motor político" [Lorenzano, 2007: 13). En las acciones, al nombrar e identificar los nombres de las víctimas, activamos la memoria del pasado en el presente, la denuncia y el reclamo a la justicia por los familiares nos liga con el futuro, convirtiendo la acción en un motor social y político. La representación reiterada y pública de los familiares alrededor del recuerdo del feminicidio evoca y exige al espectador de las acciones ser testigo y participante en la construcción del recuerdo y la denuncia.
El performance es parte integral de la plataforma Arte Acción. Entendemos el performance a varios niveles. Siguiendo el trabajo de Diana Taylor [2008],

[el] performance puede ser un objeto de análisis de 'acontecimientos como danza, teatro, rituales, protestas políticas, funerales, etc. que implican comportamientos teatrales, predeterminados o relativos a la categoría de 'evento.' También decir que algo es un performance equivale a una afirmación ontológica [Taylor, 2008: 30].

Las intervenciones públicas también son performances, en la medida en que el performance sirve no solo como una afirmación ontológica, sino también como un lente y metodología de acción. Por consiguiente, el performance también, como dice Taylor [2008], es una epistemología. El performance es un espacio de construcción de conocimiento.

Otro aspecto teórico en los estudios sobre performance es la performatividad, como lo mencionamos brevemente en la nota de pie uno de

$\nabla$ Imagen 2. Madre de niña asesinada en duelo en una acción. Fotografía: Lydia Reich.

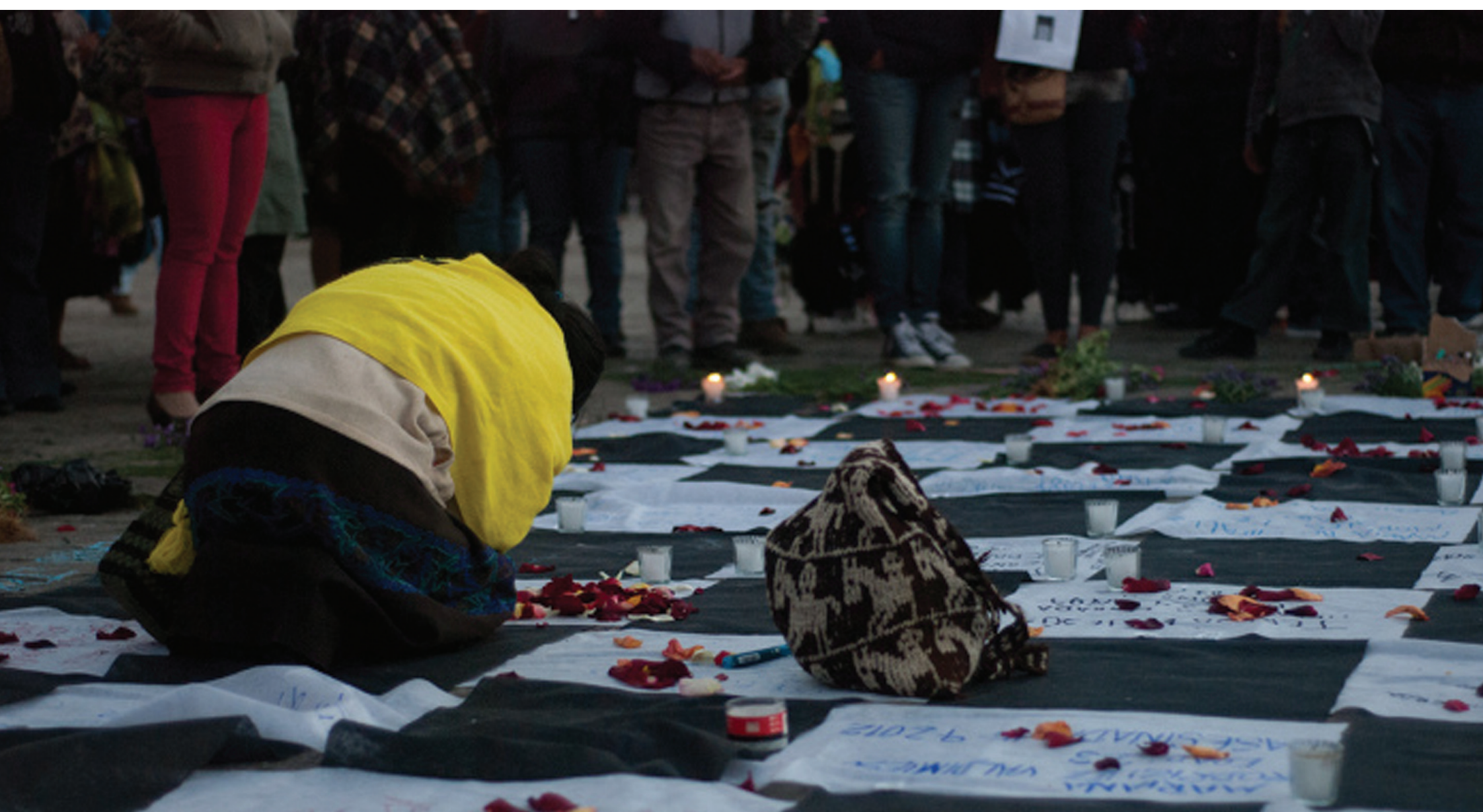




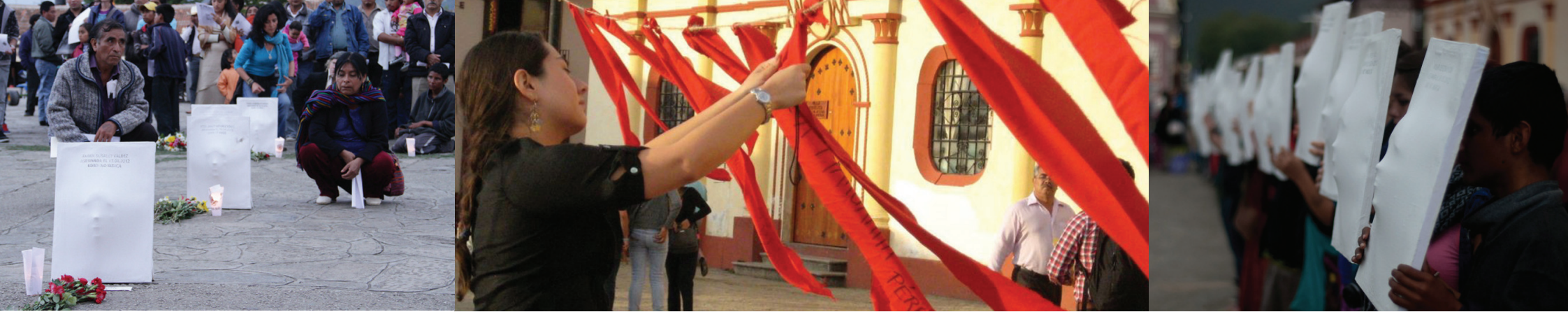

A Imagen 3. Padres de Itzel Janet Méndez en un ritual performático en la Plaza de la Resistencia. Fotografía: Lydia Reich.
A Imagen 4. Miembro de la sociedad civil que se involucró en una acción;

Fotografía: Rosy Velasco.
A Imagen 5. Participantes de la sociedad civil con epitafios de rostros de las mujeres muertas. Fotografía: Lydia Reich. la introducción. Performatividad no solo consiste en la realización de una acción con el enunciado, sino también, marca procesos de repetición de acciones producidas y controladas por prácticas regulatorias y citacionales [Taylor, 2008: 31]. Por ejemplo, el género y la sexualidad son identidades creadas por socializaciones reguladas por fuerzas normativas en una sociedad. Al repetir patrones de comportamientos que destacan y definen dichos géneros y sexualidades, se realiza un acto performativo. No siempre son actos conscientes, sino que se trata de actos internalizados y realizados sobre cuerpos. Ha sido una manera muy controversial de ver la performatividad porque reta cuestiones biológicas del cuerpo y las pone en un lugar que se construye a través de instituciones represivas y/o dominantes de poder.

Ocupar el espacio de La Plaza de la Resistencia como lugar de ritual y de resignificación de este espacio histórico en las acciones, es hacer un evento de performance. Realizar actos performativos consiste en la repetición del ritual del duelo en un espacio público [que implica una audiencia y/o testigos] y al repetir, abrir la socialización de lo que "debe" ser privado, como las emociones, la violencia impune y el duelo. Reta a la sociedad civil a reencontrarse con el espacio público, concientizándose de la realidad de la violencia feminicida en esta región de la mano de los familiares que no callan ni se esconden. El performance invita la participación activa de la "audiencia" o los testigos del evento. De esta manera, la gente alrededor o dentro de la plaza se convierte en una parte integral de cada acción.

En las acciones la cuestión política asume un rol distinto al de la "política" partidista. Entendemos lo político con Antonio Prieto Strambaugh [2008], "como una toma de postura frente a procesos que afectan la vida pública y colectiva de la sociedad; una demostración de conciencia crítica ante los impactos que estos procesos tienen en la existencia subjetiva y corporal de las personas" [Strambaugh, 2008: 609-610]. Las acciones, tanto con la participación de los familiares como con la participación de la sociedad civil, demuestran una consciencia crítica ante la impunidad y falta de procesos para penalizar la muerte violenta de estas mujeres. La política de las acciones también viene del performance como un momento creativo donde el público es interpelado. Como dice Adolfo Albán Achinte,

El acto creador asumido como una práctica deconstructiva que nos lleve a desaprender, se convierte en la posibilidad de decolonizar nuestras mentes en la medida que podamos, de la mano de la pedagogía entendida como la práctica reflexiva del sentido de ser humano, expresarnos sin miramientos ni ataduras, sin restricciones ni apocamientos y logremos sacar a flote lo que nos constriñe el alma [2013: 4].

Crear o ser creativos no es más que hurgar en las profundidades de nuestro propio ser desde donde afloran realidades que nos interpelan e interpelan nuestras propias realidades; es darnos la oportunidad de dejar descansar la rutina para enfrentar el hecho de permitirle a la imaginación que se pronuncie a favor de nuestra propia subjetividad [2013: 450).

Desde esta interpretación, abordamos un acto de decolonizar el activismo, desaprendiendo el activismo "profesionalizado" y creando un espacio abierto, performativo y creativo, donde el público 
tiene acceso libre a prácticas de reflexión. Inauguramos un espacio para criticar y profundizar los procesos actuales y hacer presente a los cuerpos desaparecidos. Cada acción es pedagógica en la medida que la repetición o la performatividad de los rituales rompen con la normalización de la violencia feminicida.

La memoria colectiva politizada es un acto pedagógico. En su mayor parte las acciones hacen referencia a formas diversas de recordar y sentir en un espacio específicamente público. La memoria colectiva recupera las emociones en contra de la amnesia social construida por la indiferencia de poderes estatales que penetran toda la ciudad. Peter Burke habla de la memoria colectiva del siguiente modo:

Con frecuencia es revelador aproximarse a los problemas desde atrás, darles la vuelta, por así decirlo. Para comprender el funcionamiento de la memoria colectiva quizá convenga investigar la organización social del olvido, las normas de exclusión, supresión o represión, y la cuestión de quién quiere que alguien olvide qué y por qué. En suma, la amnesia colectiva. Amnesia está relacionada con "amnistía", con lo que solía denominarse "actos de olvido", la supresión oficial de recuerdos de conflictos en beneficio de la cohesión social [Burke, 1999: 82].

Combatimos la amnesia con objetos específicos y simbólicos en las acciones. Utilizar objetos de ritual típicamente mexicanos como velas, flores y comida es una forma de repetir las costumbres alrededor de la fecha nacionalmente reconocida como Día de los Muertos. A través de la acción colectiva, los familiares entran en una temporalidad performativa para evidenciar la memoria de los cuerpos y la violencia.

Nos consideramos "cómplices" en lugar de "aliadas", de acuerdo con los procesos de decolonización de un grupo de activismo indígena, Medios de Acción Indígena [2014]. Ellos hacen una reapropriación del término "cómplice" para criticar el activismo típico de hoy en día, que se apropia del dolor e injusticia de los demás bajo el manto de las "alianzas." Ellos definen un cómplice como alguien que apoya la comisión de un crimen. A diferencia de lo anterior, una alianza es una solidaridad mayormente plasmada en el discurso, pero no en la acción. Muchas veces, las alianzas se basan en objetivos individuales para avanzar carreras profesionales ${ }^{3}$. La reapropriación del término "cómplice" es apropiada para las acciones que contradicen un poder estatal que ignora la violencia. Por esta razón, tales acciones son criminalizadas al nivel social y político. En el mero momento de la lucha, los cómplices se atrincheran.

El director y pensador Keniano, Ngugi Wa Thiong'o [1997], destaca el poder del performance para confrontar el poder estatal:

La guerra entre arte y el Estado es en realidad una lucha entre el poder del performance en las artes y el poder por parte del Estado; en resumen, una lucha entre representaciones del poder. El conflicto presente en las representaciones del poder se agudiza cuando el Estado se impone de forma externa, en una situación en la que existe un conquistador y un conquistado, como en el colonialismo [Wa Thiong'o, 2007: 348].

La representación de las mujeres víctimas por parte de los familiares en los espacios públicos contradice el intento de la colonización: represión y exclusión de las memorias de estos seres queridos; en su lugar, ella crea un espacio de "agencia popular" y colectiva [Vich, 2002: 4). Vich articula esta "agencia popular" como la producción de nuevos significados sociales que cambian el imaginario político [2002: 4]. Como punto de referencia, enfrentan la pérdida y la violencia de sus seres queridos, tolerada por las estructuras jurídicas, para evidenciar un imaginario social dominado por la impunidad.

Los performances visibilizan un trauma colectivo donde los familiares eligen no olvidar, reiterando, a través de la performatividad de sus acciones,

3. Para más información, véase "Abolishing the Ally Industrial Complex: An Indigenous Perspective", Disponible en inglés en <http:/ / www.indigenousaction.org/accomplices-not-allies-abolishing-the-ally-industrial-complex/>. 


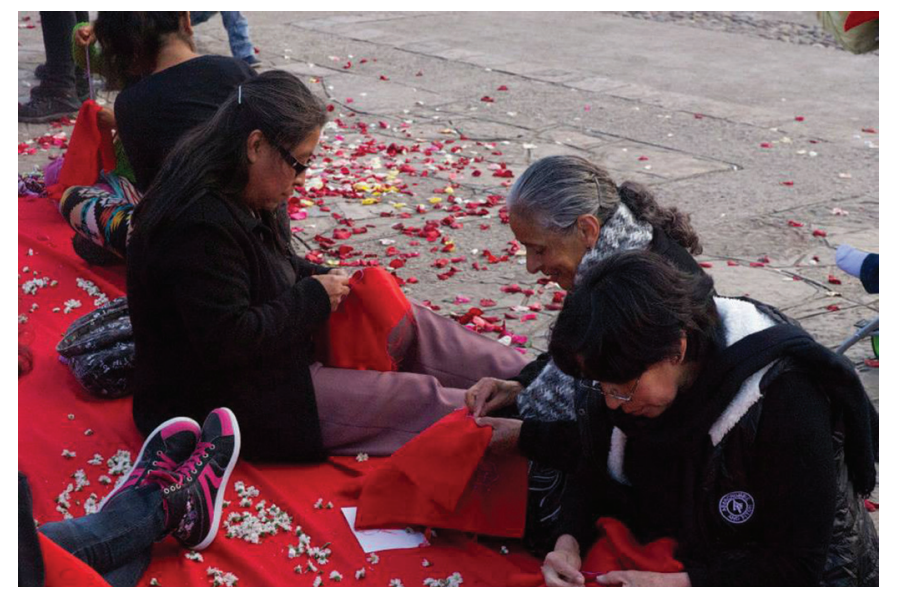

A Imagen 6. Madres bordando los nombres de sus hijas. Fotografía: Brittany Chávez.

el recuerdo de la situación de la violencia social feminicida [Taylor, 2012: 139]. En cada acción, elegimos símbolos claves para llevar la cuestión de memoria. En una de las acciones, en el Día los enamorados, utilizamos linternas con las nombres de las mujeres escritas en cada linterna. Cubrimos una calle, El Andador Guadalupe, con las linternas. Las linternas fueron colocadas en el andador guadalupano con la intención de enfatizar la violencia

$\nabla$ Imagen 7. Linternas en el Andador Guadalupe. Fotografía: Grace Remington.

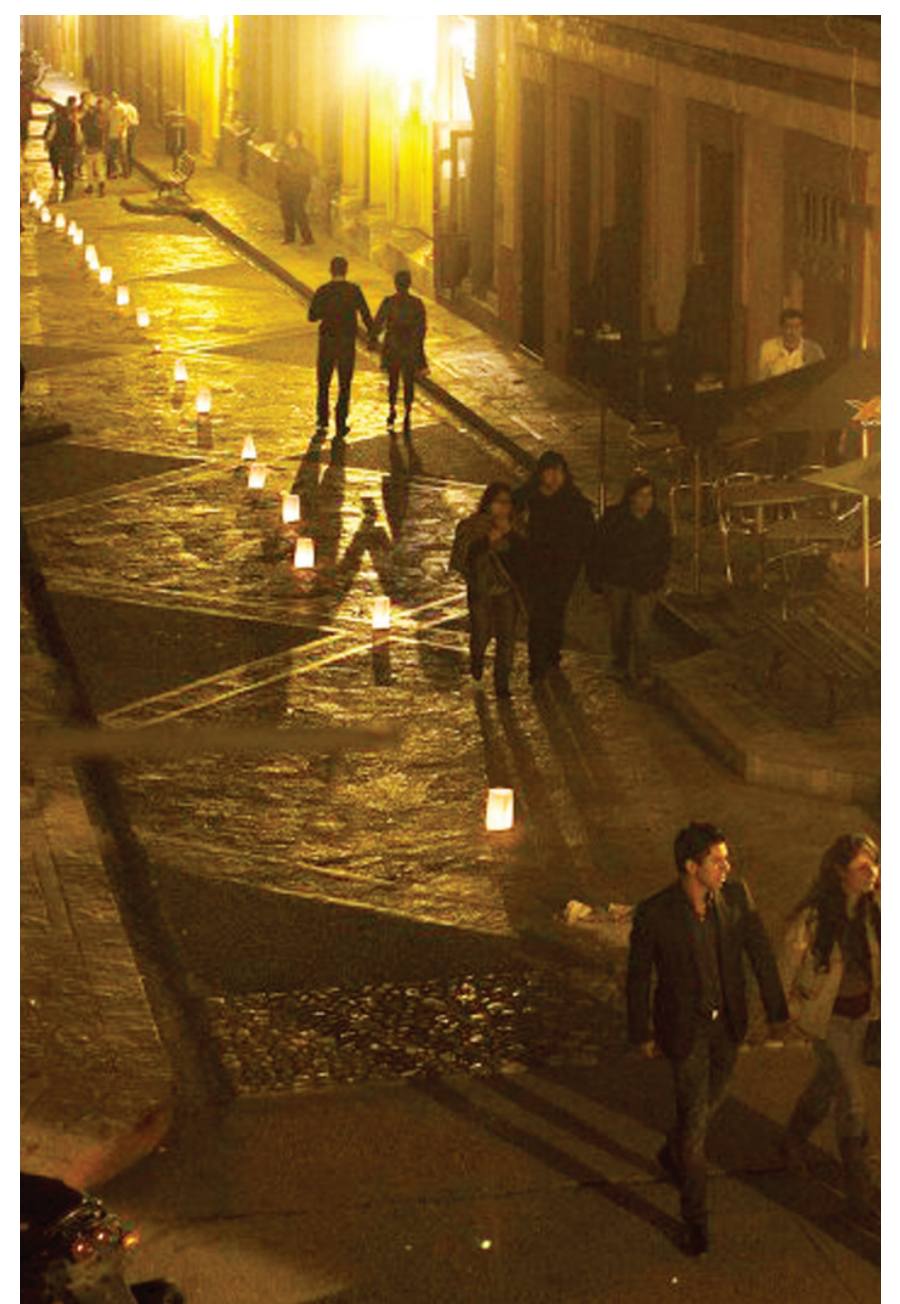

en un día típicamente asociado con el amor y el consumo desmedido. También queríamos hacer presente el hecho de que la mayor parte de los feminicidios son resultado de la violencia de pareja. En otra acción, utilizamos globos de papel de aproximadamente un metro de alto, con las nombres de las mujeres y sus edades escritos en papeles que colgaban de los globos. Los globos se levantan hacia el cielo al prender una mecha ubicada en la parte inferior. Había 66 globos representando las 66 mujeres que habían muerto por causa de la violencia feminicida en el año 2013.

Cada globo fue encendido por los participantes de la sociedad civil y fueron soltados una vez que se calentaron suficientemente para subir. Mientras más tiempo pasaba, más gente se reunía en la plaza con el fin de participar. Al final, ya no había globos pero la gente seguía pidiendo y celebrando las luces, que al subir al cielo iban revelando los nombres.

En esta acción vimos la memoria volar. Las mujeres se convirtieron en luces de la noche, subiendo

$\nabla$ Imagen 8. Globos subiendo en la Plaza Presidencial en San Cristóbal. Fotografía: Moysés Zúñiga Santiago.

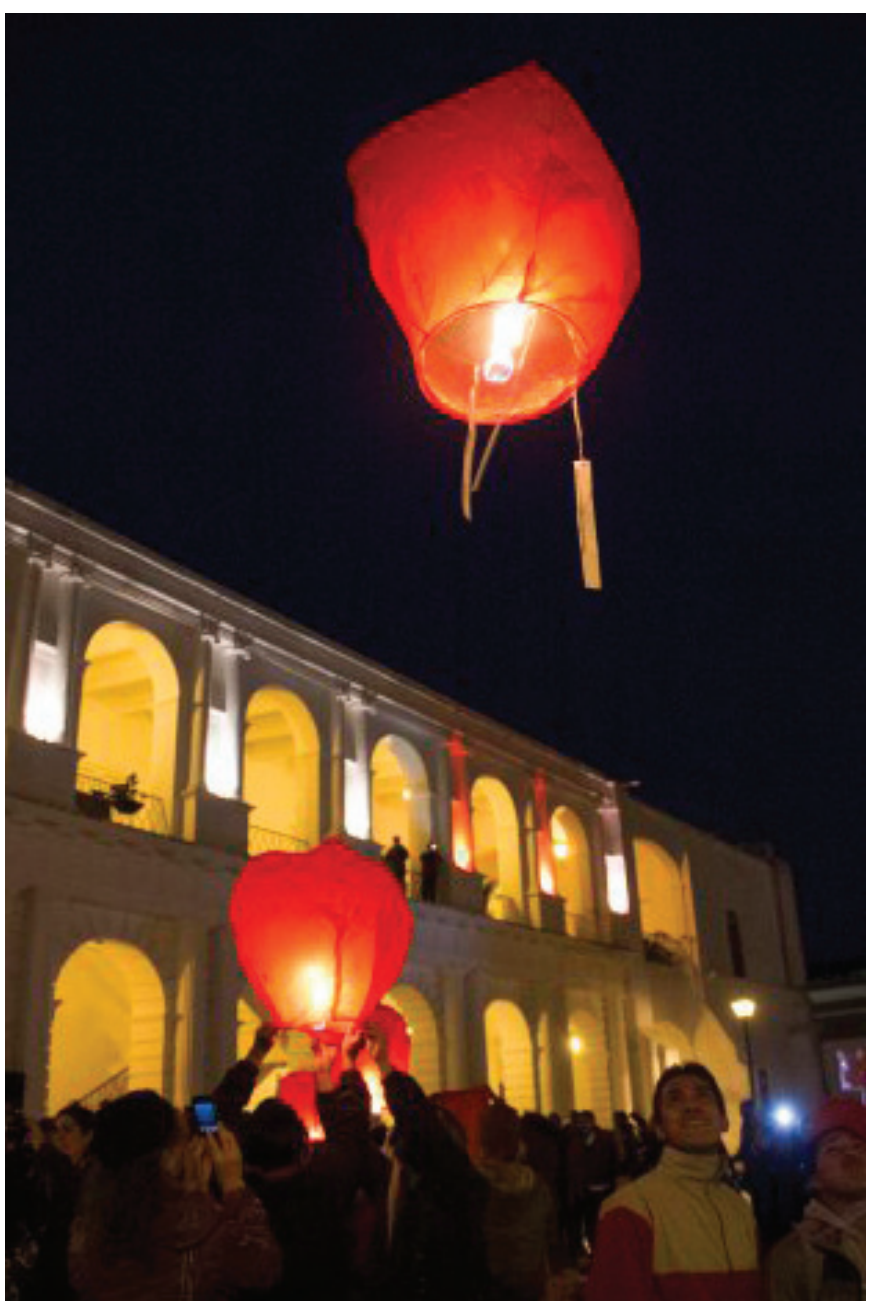


más arriba de la Plaza Presidencial, mientras los guardias, desde sus puestos, miraban con ojos de sospecha e interés. En una de las acciones más recientes, en colaboración con un grupo de México D. F. que borda imágenes de feminicidios en superficies de tela y algunos de los familiares, ocupamos todos la Plaza de la Resistencia por varias horas, bordando los nombres de las mujeres e invitando la participación de los transeúntes. Varios niños artesanos pararon su trabajo y participaron en la acción, bordando con las madres de las mujeres asesinadas. [Imagen 9].

Todas estas acciones/performances son momentos pedagógicos de activismo decolonial. Involucrando cuerpos de la ciudad, manejamos el espacio público como sitio de lucha.

Es muy posible que surja la palabra "feminismo" dentro de estas acciones políticas. Como lo señala Rosalva Aída Hernández Castillo, es un momento clave para construir diálogos más constructivos entre los feminismos poscoloniales y decoloniales (Hernández Castillo, 2008: 78). De acuerdo con nuestro posicionamiento pedagógico-decolonial, las acciones políticas performativas nos llevan a otro diálogo, a otra elaboración de la narrativa sobre la violencia feminicida que transmite y denuncia desde las familias, la reflexión y el debate; que en ausencia del Estado y un sistema judicial, registra y problematiza la realidad. Las acciones son un motor político que inicia desde la sociedad civil, ejerciendo la memoria y el no olvido como formas de resistencia. La práctica decolonial performativa y pedagógica de las acciones va en contravía de las normas regulatorias del poder opresivo que instala la "amnesia" selectiva en la sociedad civil.

\section{Re-existiendo y caminando: una plata- forma de praxis y teoría para seguir andando}

Xochitl Leyva Solano [2010] describe otra forma de pensar un andar decolonial: "[...] las prácticas decoloniales son un proceso que se va construyendo, poco a poco, colectivamente desde la práctica, desde abajo, desde lo personal, lo político, lo espiritual, lo subjetivo e intersubjetivo"

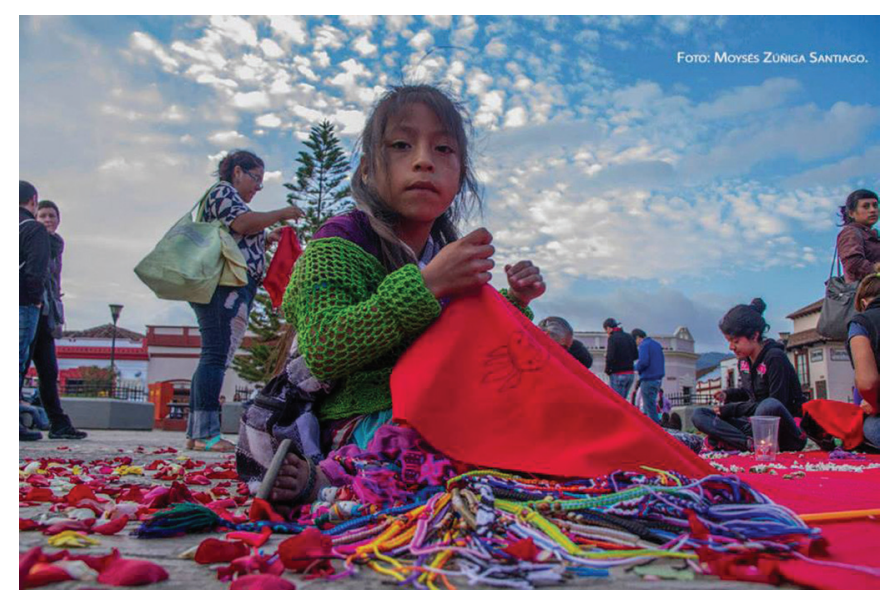

A Imagen 9. Niña bordando en la Plaza de la Resistencia. Fotografía: Moysés Zuñiga Santiago.

[Solano, 2010: 370]. Arte Acción funciona como una plataforma para arte en resistencia. Como mencionamos en la introducción, no solo abordamos el tema del feminicidio, sino que también utilizamos herramientas performativas para abordar otros temas. Recientemente realizamos una colaboración basada en la concepción del mapa antioccidental, con el Sur arriba. En un performance colaborativo de larga duración, Brittany Chávez se tatuó el mapa de las Américas invertido en la espalda ante un público íntimo en un espacio radical, El Paliacate Espacio Cultural, acompañada de fotografías de Moysés Zuñiga Santiago del tren La Bestia [donde miles de migrantes cruzan la frontera entre Guatemala y Chiapas sobre el tren] y poesía de Iván Darío Vargas Roncancio. Este performance marca otra forma de resistir los poderes coloniales que dominan cuerpos y existencias.

Decidimos armar este performance bajo la plataforma de Arte Acción para seguir con la política de resignificar el cuerpo en el espacio público y el cuerpo dentro de su propia piel. Utilizar el cuerpo de esta manera aborda una geopolítica que nos lleva a construir otras formas de convivir, ser, estar y sentir dentro de espacios históricamente determinados y marcados. Este performance, Otredades: Cartografía Corporal 1.0, no se trata de un cuerpo femenino que elige ser tatuado ante o para un público voyerístico. Tampoco se trata de un simple hecho estético o una prueba de aguante del dolor. A pesar de que la cuestión estética está presente en lo visual de la acción y el dolor muy presente a raíz de las agujas sobre la piel por dos horas sin parar, el performance 
activó un espacio para pensar las fronteras geográficas y corporales donde la violencia se expande y se convierte en amnesia social.

El calor del cuerpo humano presente en una acción pública en tiempo real insiste en un sentir donde esferas energéticas, somáticas, espirituales, tangibles y multisensoriales dibujan y controlan el espacio del performance. En este espacio las líneas entre la realidad y el tiempo performativo se entretejen y abren un espacio potencial de re-existencia: ningún cuerpo experimenta la acción de la misma manera y para cada cuerpo la experiencia corporal se registra de una manera distinta. Durante el performance, la audiencia/testigos fueron invitados a tocar los brazos y las piernas de la performera para transmitir energías o dar calma, con el fin de suavizar la experiencia del dolor y para sentir las varias posibilidades de transmisión entre cuerpos. Algunas personas se quedaron durante las dos horas, otras se quedaron poco tiempo pero dejando saber a la performera que estaban presentes durante el proceso de la corporalización del mapa y compartiendo la experiencia física y espiritual.

Esta acción marcó un cambio en la amplitud de temas que abordamos en la plataforma Arte Acción. El mapa invertido lucha en contra de la noción del Norte siempre-arriba y también resignifica la inversión no como un movimiento retrasado o hacia atrás, sino deseado. El mapa, inscrito de una manera permanente en el cuerpo, señala varias rutas que han marcado historias de cruce, de violencia, de pérdida, de olvido y de territorios separados por políticas nacionales e internacionales. Nos habla de genocidios, feminicidios, rutas de tráfico de gentes, migraciones peligrosas y/o forzadas y demás. Como primera instalación en una serie, este performance sirvió para marcar un proceso de territorialización y reterritorialización, abrazando la incapacidad de desconectarnos completamente del patrón colonial histórico del movimiento de seres humanos, aunque intentamos ir hacia un re-existir en un andar decolonial.

Este activismo corporal decoloniza el cuerpo por medio del performance y trabaja de una manera pedagógica al abrir espacios a otras formas de sentir y pensar. Es un activismo que hace teoría vivida y corporal performativamente. El performance empieza citando feminicidios desde el lugar donde ocurren -el cuerpo- y expande el contexto de los espacios corporales que funcionan como excusa o raíz de la extrema violencia. Es una forma de teorizar sobre cómo liberamos el cuerpo o cómo quisiéramos que fuera liberado. El mapa, en el Estado colonial occidental, representa una esclavitud impuesta por fronteras y divisiones que limita el flujo libre de gentes. Entonces, al marcarlo de una manera permanente, suele repetir un patrón colonial en lugar de luchar en contra. Desde ahí, entra el activismo decolonial pedagógico del performance que voltea poderes impuestos al luchar a través del único campo de batalla que nos queda: el cuerpo. Este activismo

V Imagen 10 Performance de Brittany Chávez, "Otredades: Cartografía Corporal 1.0.” Fotografía: Moysés Zúñiga Santiago.

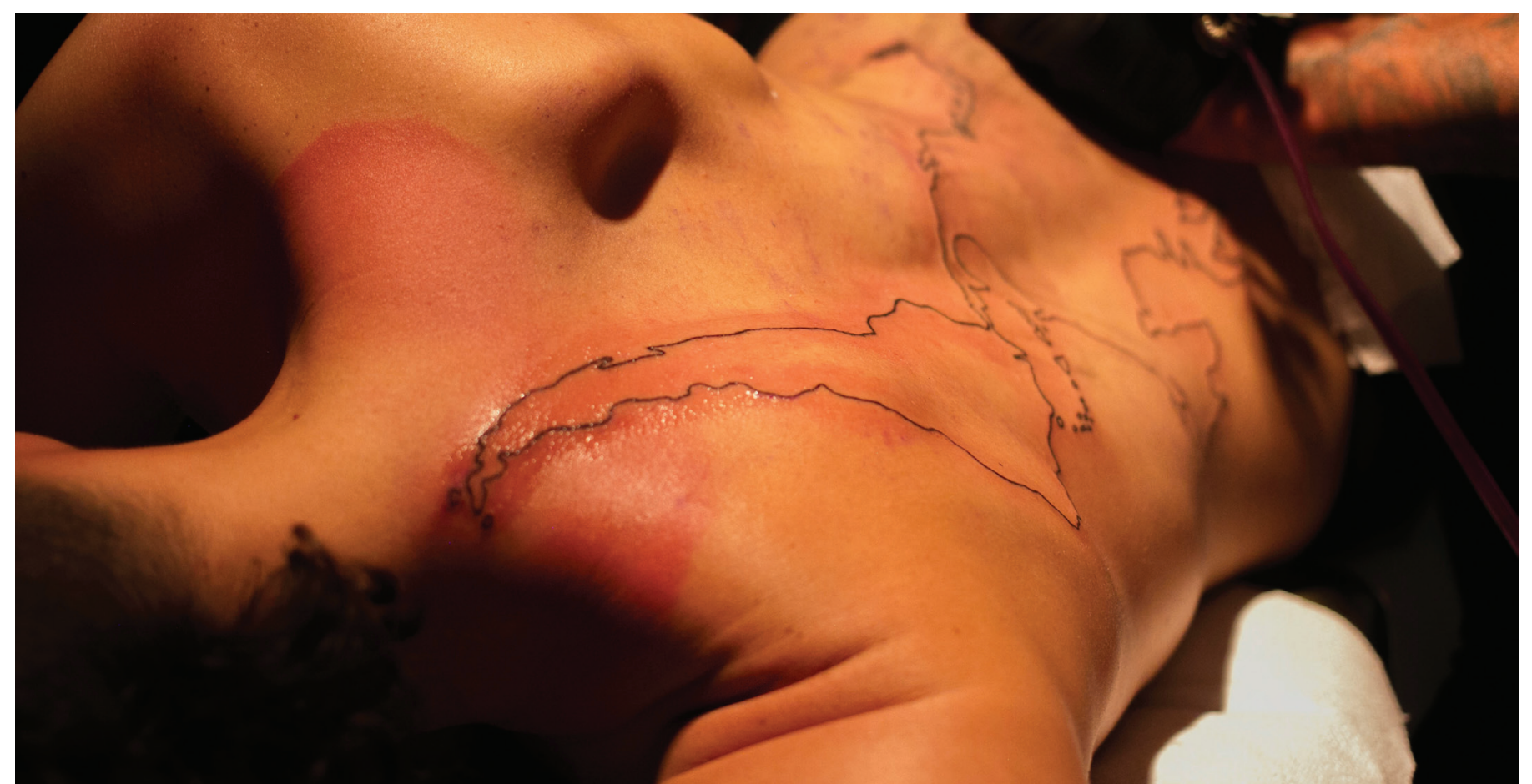


señala que la realidad de fronteras y divisiones geográficas es inventada, y forma parte de una geografía corporal para seguir resignificando e interviniendo.

En cada acción, regresamos a lo erótico, lo decolonial y lo pedagógico. Es un tejido que construye un mapa activista que nos dirige a otras formas de actuar y resistir. Esperamos que una plataforma que utiliza el performance como un método decolonial pedagógico pueda abrir otros espacios de diálogo activista. Regresamos al cuerpo en cada instante. Como nos enseña Lorde, "Reconocer el poder de lo erótico en nuestra vidas nos puede dar la energía para perseguir cambios genuinos en nuestro mundo, en vez de ser cómplices de los mismos personajes en el mismo drama desgastado" [1979: 59]. Mientras que nuestro enfoque ha sido el feminicidio, a partir de ahora incluimos otras luchas de cuerpos disidentes. Es a través de Arte Acción, con su enfoque en performance y performatividad, que seguimos rompiendo esquemas y patrones de poder.

\section{Referencias}

Albán Achinte, Adolfo [2013]. "Pedagogías de la re-existencia. Artistas indígenas y afrocolombianos", en Catherine Walsh [ed.], Pedagogías decoloniales: Prácticas insurgentes de resistir, (re) existir y (reJvivir. Quito: Abya-Yala Quito: Ediciones Abya Yala.

Burke, Peter [1999]. Formas de Historia Cultural. España: Alianza Editorial.

Enciso, Graciela Reyermuth [2008). "Realidad y disimulo: complicidad e indiferencia social en Chiapas frente a la muerte femenina", en Mercedes Olivera, Violencia feminicida en Chiapas: Razones visibles y ocultas de nuestras luchas, resistencias y rebeldías. Tuxtla Gutiérrez, Chiapas, México: Colección Selva Negra, UNICACH.

Figueroa Mier, Martha Guadalupe [2008]. "Violencia feminicida e impunidad en Chiapas", en Mercedes Olivera, Violencia Feminicida en Chiapas: Razones visibles y ocultas de nuestras luchas, resistencias y rebeldías. Tuxtla Gutiérrez, Chiapas, México: Colección Selva Negra, UNICACH.
Hernández Castillo, Rosalva Aída [2008]. "Feminismos poscoloniales: reflexiones desde el sur del Río Bravo", en Liliana Suárez Navaz y Rosalva Aída Hernández [eds.], Decolonizando el feminismo: teorías y prácticas desde los márgenes. Madrid: Instituciones Cátedra. Indigenous Action Media [2014]. "Abolishing the Ally Industrial Complex: An Indigenous Perspective". Disponible en inglés en <http:/ / www.indigenousaction.org/accomplices-not-allies-abolishingthe-ally-industrial-complex/>

Lagarde, Marcela [1997]. "Identidades de género y derechos humanos. La construcción de las humanas." Ponencia presentada en el VII Curso de Verano. Educación. Democracia y nueva ciudadanía. Universidad Autónoma de Aguascalientes.

Lorde, Audre [1978]. "Uses of the Erotic: The Erotic as Power", en Audre Lorde, Sister Outsider: Essays and Speeches by Audre Lorde. Berkeley: Crossing Press.

Lorenzano, Sandra [2007]. "No aportar silencio al silencio. A modo de introducción”, en Políticas de la Memoria: Tensiones en la Palabra y la Imagen. Argentina: Editorial Gorla.

Marcos, Subcomandante Insurgente [2014]. "Entre la luz y la sombra". Disponible en <http:/ / enlacezapatista.ezln.org.mx/2014/05/25/ entre-la-luz-y-la-sombra/>

Olivera, Mercedes [2008]. "Violencia feminicida en México: expresión de la crisis estructural", en Mercedes Olivera, Violencia feminicida en Chiapas: Razones visibles y ocultas de nuestras luchas, resistencias y rebeldías. Tuxtla Gutiérrez, Chiapas, México: Colección Selva Negra, UNICACH.

[2011]. Mujeres marginales de Chiapas: situación, condición y participación. Tuxtla Gutiérrez, Chiapas, México: Universidad de Ciencias y Artes de Chiapas.

Osuna, Mercedes [2013]. "Aumenta el feminicidio en Chiapas", en des/nformémonos: periodismo de abajo, No. 115. Disponible en <http:// desinformemonos.org/2014/05/aumenta-elfeminicidio-en-chiapas/> 
Segato, Rita Laura [2010]. "Género y colonialidad en busca de claves de lectura y de un vocabulario estratégico descolonial." en Quijano, Aníbal y Julio Mejía Navarrete [eds.], La Cuestión Descolonial. Lima: Universidad Ricardo Palma - Cátedra América Latina y la Colonialidad del Poder.

Solano, Xochitl Leyva [2010]. "Caminando y haciendo o acerca de prácticas decoloniales", en Axel Köhler et. al., Sjalel kibeltik. Sts'isjel ja kechtiki'. Tejiendo nuestras raíces. México, D.F: RACCACH, Cesmeca-Unicach, CIESAS, PUMCUNAM, IWGIA, Orê y Xenix Filmdistribution.

Stambaugh, Antonio Prieto [2008). "Corporalidades políticas: representación, frontera y sexualidad en la performance mexicana", en Diana Taylor y Marcela Fuentes, Estudios avanzados de performance. México, D.F.: Fondo de Cultura Económica.

Taylor, Diana [2008]. "Hacia una definición de performance", en Paolo Vignolo [ed.], Ciudadanías en escena: performance y derechos culturales en Colombia. Bogotá: La Cátedra Manuel Ancízar.

[2012]. "Yuyachkani: trauma y memoria en escena", en Diana Taylor, Acciones de memoria: performance, historia, y trauma. Lima: Fondo Editorial de la Asamblea Nacional de Rectores.

Trigo, Abril [2007]. "Entre la globalización de la memoria y las memorias de la globalización [Apuntes]", en Sandra Lorenzano y Ralph Buchenhorst [eds.], Políticas de la memoria: tensiones en la palabra y la imagen. México, D.F.: Editorial Gorla, Universidad del Claustro de Sor Juana.

Vich, Víctor [2002]. "Introducción”, en Víctor Vich, El discurso de la calle. Los cómicos ambulantes y las tensión de la modernidad en Perú. Perú: IEP Instituto de Estudios Peruanos.

Walsh, Catherine [2013]. "Introducción: Lo pedagógico y lo decolonial: Entretejiendo caminos”, en Catherine Walsh [ed.], Pedagogías decoloniales: Prácticas insurgentes de resistir, (relexistir y (re) vivir. Quito: Ediciones Abya Yala.

Wa Thiong'o, Ngugi. [1997]. "Actuaciones del poder: la política del espacio de performance", en
Diana Taylor y Marcela Fuentes, Estudios avanzados de performance. México, D.F.: Fondo de Cultura Económica Carretera Picacho-Ajusco. 